\title{
REFORMA DE EDIFICIOS ANTIGUOS. EL RASGADO DE HUECOS EN MUROS DE CARGA
}

\author{
(OPENINGS BREACKAGE IN LOAD-BEARING WALLS)
}

M. Soto Pardo,

Dr. Ingeniero ICAI

Director de "Soto Pardo, S. A.». Empresa constructora

\section{RESUMEN}

La apertura de huecos en muros de carga es una operación frecuente en la reforma de edificios antiguos. Una correcta ejecución exije la puesta en carga de la estructura sustentante - normalmente un pórtico metálico- mediante gatos hidráulicos o algo similar, cosa que suele omitirse, dando lugar a dos problemas diferentes: uno, el desprendimiento diferido del área de descarga, con alarmantes fisuraciones y otro, sin efectos ostensibles, pero mucho más peligroso, como es la sobrecarga elevadísima en ciertas zonas de la fábrica que puede desembocar en un colapso por aplastamiento. Este artículo estudia y cálcula la puesta en carga para una correcta ejecución del rasgado. También se ofrece un método, original, de estimación de calidades resistentes de las fábricas de ladrillo a rasgar, efectuando "in situ", que complementa las apreciaciones visuales.

\section{SUMMARY}

Forming openings in load-bearing brickwork is a very frequent operation when reforming old buildings. A correct solution requires the supporting structure, usually a steel portal frame, to be loaded by means of hydraulic jacks or some similar system. This step is normally omitted which gives rise to two different problems. Fistly, a delayed loosening of the unsupported area occurs and alarming cracks appear. Secondly and much more dangerous though not so apparent, is the overloading of certain areas of brickwork which can result in collapse due to bearing failure. This article considers the loading sequence for correctly opening up the brickwork. It also provides an original in situ method for estimating brickwork strengths as a complement to a visual inspection.

\section{INTRODUCCION}

La figura 1 muestra una fachada afectada por una serie de grietas ocasionadas por la ejecución de un rasgado de hueco para una instalación comercial en la planta baja. Cualquier observador que se dé una vuelta por el casco viejo de una ciudad podrá detectar, con un poco de ojo clínico, fisuras similares a éstas, coincidiendo casi siempre con una instalación comercial en los bajos de un edificio antiguo dotado de fachada resistente. Se trata de las "reliquias» o cicatrices de una cirugía mai ejecutada. Esta cuestión es especialmente importante ahora, en que la rehabilitación de edificios cobra especial auge, siendo necesario diafanizar a veces muros de carga en crujías interiores.

Mi propósito es arrojar alguna luz sobre un tipo de trabajo tan frecuentemente acometido como deficientemente ejecutado. No es que el trabajo se haga mal, sino que se hace incompleto.

El calculista diseña un dintel de acuerdo con las cargas contenidas en la parábola de descarga o el rectángulo (Fig. 2), según $M V-201-1972$. Si se trata de una fachada con huecos, el área de descarga se ampliará en sentido vertical hasta la altura total del edificio, ya que los huecos propagan la línea de rotura hacia arriba, como muestra la figura 3 . En este caso el dintel resultará, naturalmente, un perfil mucho más importante. Aquí termina, de ordinario, el proyectista; a lo sumo, sobredimensionará el cargadero si el cálculo le da un perfil pequeño.

Puestos a ejecutar el trabajo, se procede primero al apeo del hueco, se rasga el mismo, se introduce el cargadero, se retaca el espacio entre el ala superior del mismo y la fábrica y se quitan los puntales al final.

Así las cosas, es evidente que el muro «no se ha enterado" de la colocación del cargadero. El flujo de isostáticas de compresión no ha variado; continúa como en la figura 2. La razón es que el dintel colocado no ha entrado en carga, pues para ello se precisa una cierta deformación en el mismo. He aquí el error: no poner en carga el perfil.

La historia de lo que viene a continuación es siempre la misma. La zona de descarga comienza lenta- 


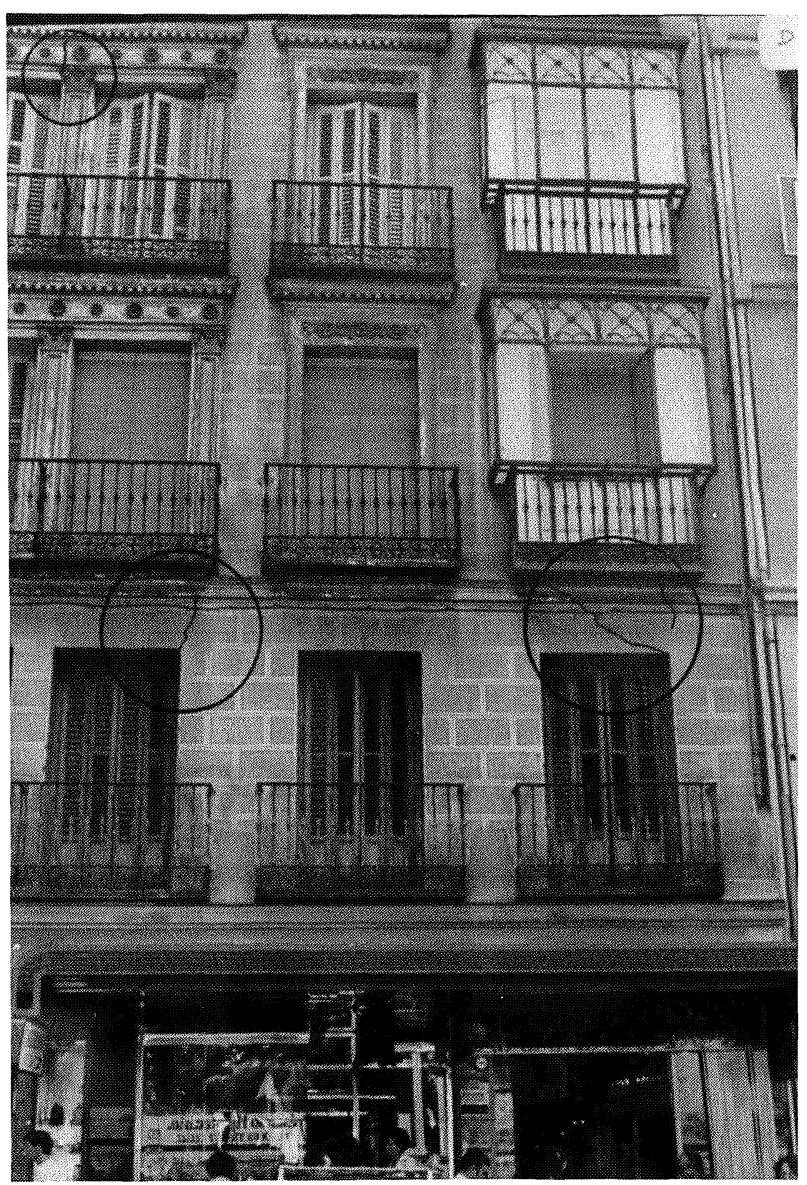

Fig. 1.-Fachada resistente fisurada por la apertura de un hueco comercial en planta baja sin la preceptiva puesta en carga del dintel.

mente a descender, asentándose sobre el cargadero, en un proceso que puede durar varios años, según la calidad de la fábrica, luz del hueco y, sobre todo, las vibraciones que reciba, bien por uso, bien por tráfico rodado cercano.

Tan pronto aparecen las primeras fisuras, surge la alarma en el vecindario y comienzan las conversaciones con los «culpables»-propiedad y dirección técnica - los cuales han de admitir la evidencia de que antes de su obra de rasgado no había grietas. Al vecindario se le suele tranquilizar diciendo que «esto es normal» y se colocan de ordinario unos testigos o bien se tapan las grietas y se pinta. Como quiera que el proceso sigue, a los pocos meses las grietas se vuelven a abrir, si bien, con menos intensidad que al principio.

No hace falta decir que, en la situación que describo, han intervenido ya notarios, abogados, etc., y que el técnico responsable ha tenido que emitir algún certificado de seguridad del edificio, redactado más o menos ambiguamente.

Hay que decir que la batalla suele endurecerse cuando la propiedad de los bajos es económicamente fuerte $y$ "hay de donde sacar». Es el caso típico de los Bancos. El «affaire» llega a terminar al

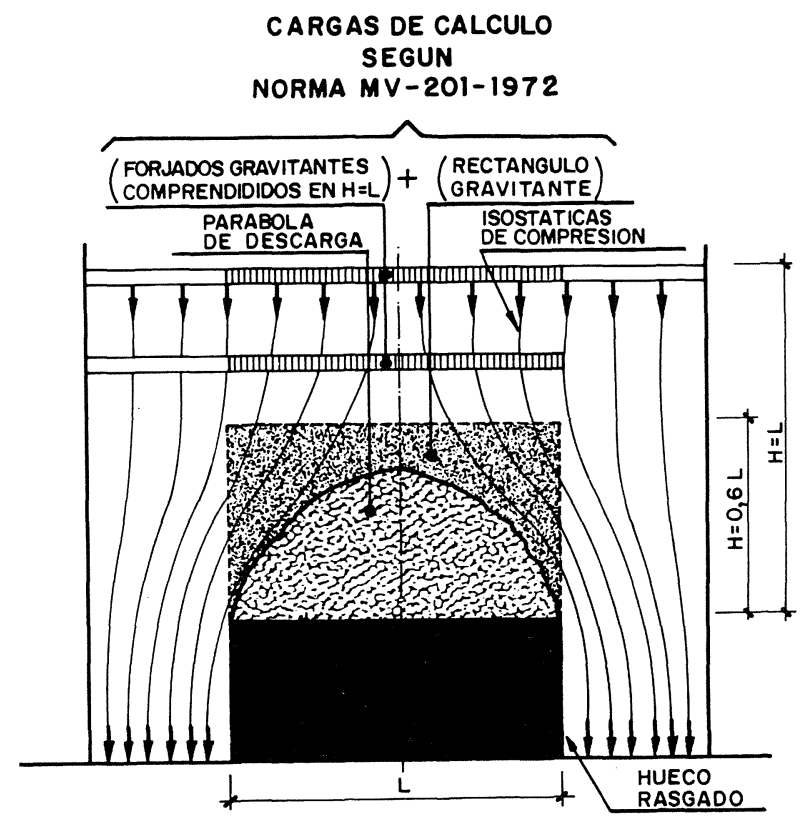

Fig. 2.-Cargas que actúan en el rasgado de un muro sin huecos. Flujo de líneas de compresión.

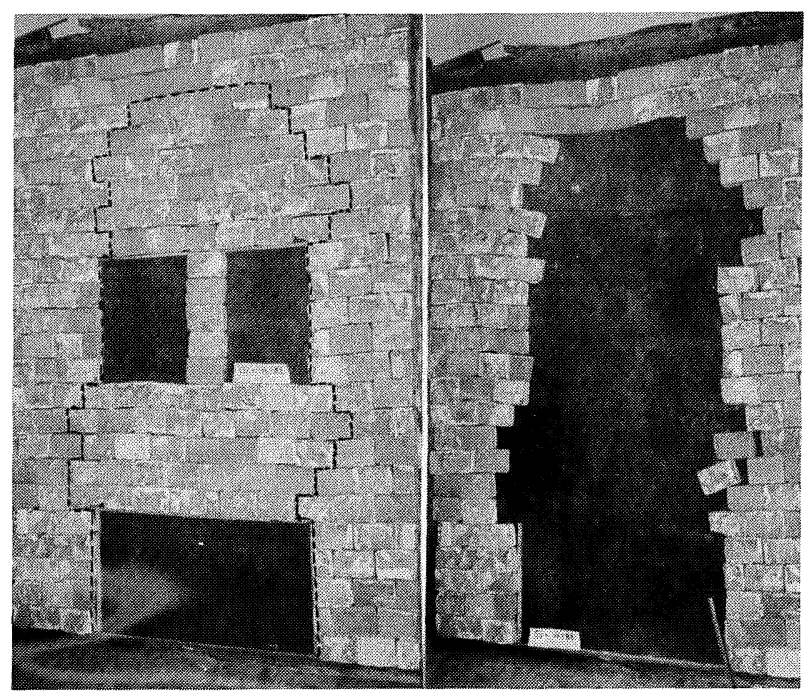

Fig. 3.-Deformación del arco de descarga parabólico por la presencia de huecos en el muro. Ensayo a escala reducida.

cabo de meses o años. Las grietas se estabilizan. No vuelven a salir después de unas cuantas fases de retoques y pinturas. El arco de descarga ha llegado por fin a su sitio: se ha descolgado totalmente sobre el dintel, el cual ha tomado su deformación correspondiente. $\mathrm{Ha}$ entrado en carga.

En la figura 1 se han reforzado a tinta las fisuras existentes para su mejor visibilidad. Ello no falsea la realidad, ya que estas fachadas han sido objeto de varios «maquillajes» y las grietas residuales aparentes no representan la realidad.

Durante el período descrito tienen lugar dos episodios netamente distintos y que conviene diferenciar: 
a) La fisuración del muro resistente por descenso del área de descarga, de efectos llamativos, pero de peligrosidad prácticamente nula.

b) La sobrecarga de las pilastras laterales del hueco rasgado (ver la concentración de isostáticas en la figura 2), cuyo material puede alcanzar tensiones de compresión altísimas y llegar al colapso. De esto nadie se percata porque no hay síntomas externos. Tengo para mí, que el hundimiento de un edificio en Madrid, hacia los años 70, en el que se ejecutaba una instalación comercial en los bajos, pudo deberse a este fenómeno. No cito la ubicación por falta de pruebas claras.

¿Se puede evitar de alguna forma tanto el calvario a que conduce el fenómeno a) como el peligro latente que se desprende de b)? La respuesta es afirmativa. No hay más que poner en carga el dintel al rasgar el hueco y, en su caso, reforzar la pilastra.

\section{PUESTA EN CARGA DEL DINTEL}

Consiste esta operación en obligar al dintel -mediante gatos hidráulicos, generalmente- a adquirir la deformación que le producirá la carga de cálculo una vez desprendida. Con ello crearemos unas reacciones dintel-fábrica que «sostengan» permanentemente el área de descarga, evitando todo descenso de ésta y, al propio tiempo, transmitan a los apoyos de aquél las compresiones que, de otro modo, se canalizarían a través de las pilastras laterales. Todo ello se ampliará en las líneas que siguen.

\section{RESISTENCIA DEL MURO}

Los muros a rasgar pueden estar formados por una fábrica de gran calidad y resistencia o por un entramado deleznable de cascote y madera, incluso por adobe. En estos últimos el rasgado es difícil ya desde la primera operación, el apeo, al no admitir las cargas concentradas del apuntalamiento. La operación es tanto más fácil y segura cuanto mayor es la calidad del material que forma el muro. Un primer paso es conocer la resistencia a compresión de éstos. Nos vamos, en lo siguiente, a referir a fábricas de ladrillo por fijar ideas, pero lo que se diga de éstas es aplicable a otras, como mampostería.

\section{Fábricas de ladrillo}

La Norma MV-201-1972 permite la estimación de la resistencia de cálculo de diversas fábricas. Hay que distinguir:

1) Resistencia característica a compresión $\sigma_{k}$.

2) Resistencia de cálculo $\sigma^{*}=\frac{\sigma_{\mathrm{k}}}{\gamma_{\mathrm{m}}}$ o resistencia minorada $\left(\gamma_{\mathrm{m}}=2,5\right)$
3) Resistencia afectada de minoración por pandeo:

$$
\sigma_{\mathrm{p}}^{*}=\frac{\sigma^{*}}{\gamma_{\lambda}}
$$

dependiendo $\gamma_{\lambda}$ de la esbeltez de la pieza comprimida.

4) Acciones mayoradas $N^{*}=\gamma_{s} \cdot N$, siendo $N$ la carga de servicio y de ordinario $\gamma_{m}=1,65$.

5) Fatiga o carga unitaria admisible:

$$
\sigma_{\mathrm{ad}}=\frac{\sigma_{\mathrm{k}}}{\gamma_{\mathrm{m}} \cdot \gamma_{\mathrm{s}} \cdot \gamma_{\lambda}}
$$

Para el término de pandeo $\gamma_{\lambda}$ podemos tomar (en el caso común de fábrica arriostrada por forjado):

$\begin{array}{lll}\lambda=5 & 10 & 15 \\ \gamma_{\lambda}=1,25 & 2,00 & 3,33\end{array}$

Una primera orientación sencilla y concisa nos puede conducir al siguiente cuadro:

\begin{tabular}{|l|r|r|r|r|}
\hline & \multicolumn{5}{|c|}{$\sigma_{\text {ad }}$ en $\mathrm{kg} / \mathrm{cm}^{2}$ para una esbeltez $\lambda=$} \\
\multicolumn{1}{|c|}{ Tipo de fábrica } & \multicolumn{1}{c|}{0} & \multicolumn{1}{c|}{10} \\
\hline Fábricas muy antiguas & & & & \\
de ladrillo macizo & 7,00 & 5,60 & 3,50 & 2,10 \\
Fábricas antiguas (50 & & & & \\
años) id. id. & 10,00 & 8,00 & 5,00 & 3,33 \\
Fábricas modernas id. & 30,00 & 24,00 & 15,00 & 10,00 \\
Id. id. ladrillo hueco & 8,00 & 6,40 & 4,00 & 2,40 \\
\hline
\end{tabular}

\section{Estimación de resistencia por carga concentrada}

La figura 4 ilustra sobre un procedimiento aproximado para obtener un valor estimativo de la resistencia de la fábrica que tenemos entre manos. Se

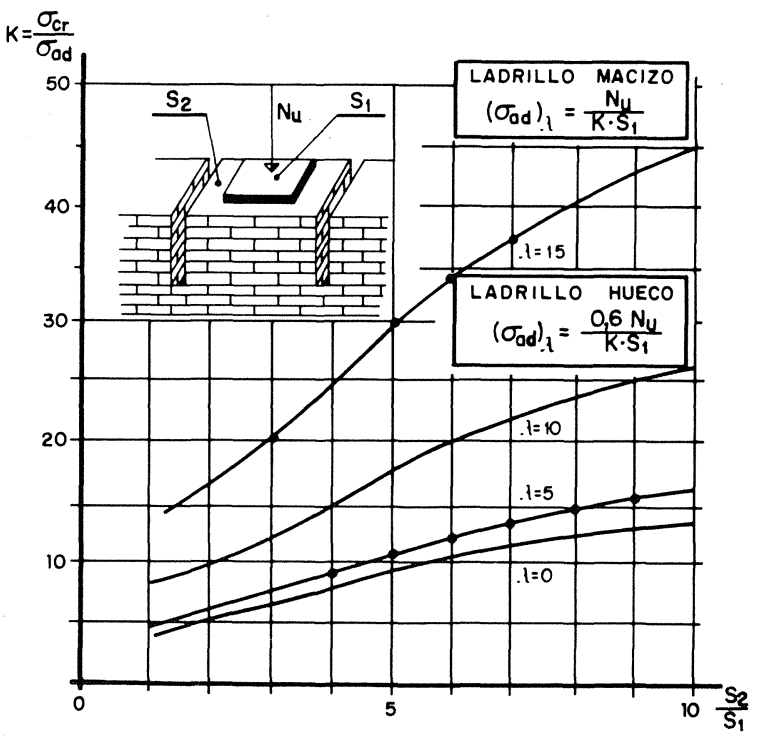

Fig. 4-Correlación entre la carga de rotura concentrada $\left(N_{u}\right)$, la admisible $\left(\sigma_{a d}\right)$ y la de relación de concentración $\left(S_{2}: S_{2}\right)$ en función de la esbeltez de la pieza $\lambda$ 


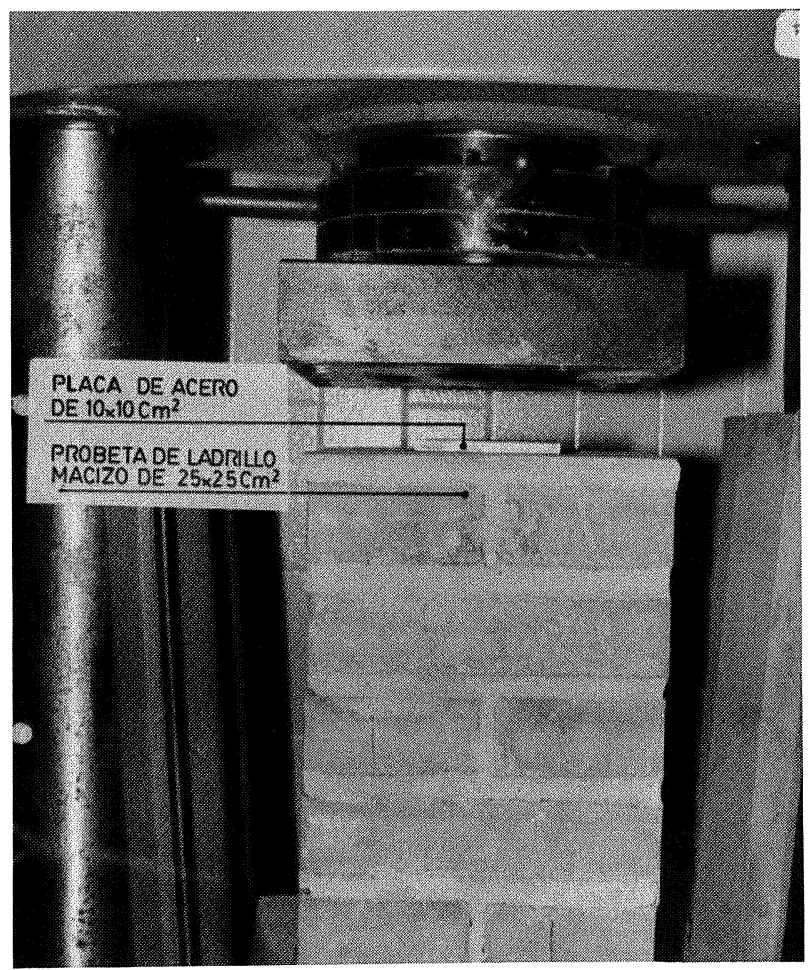

Fig. 5.-Ensayos para determinación de curvas de figura 4.

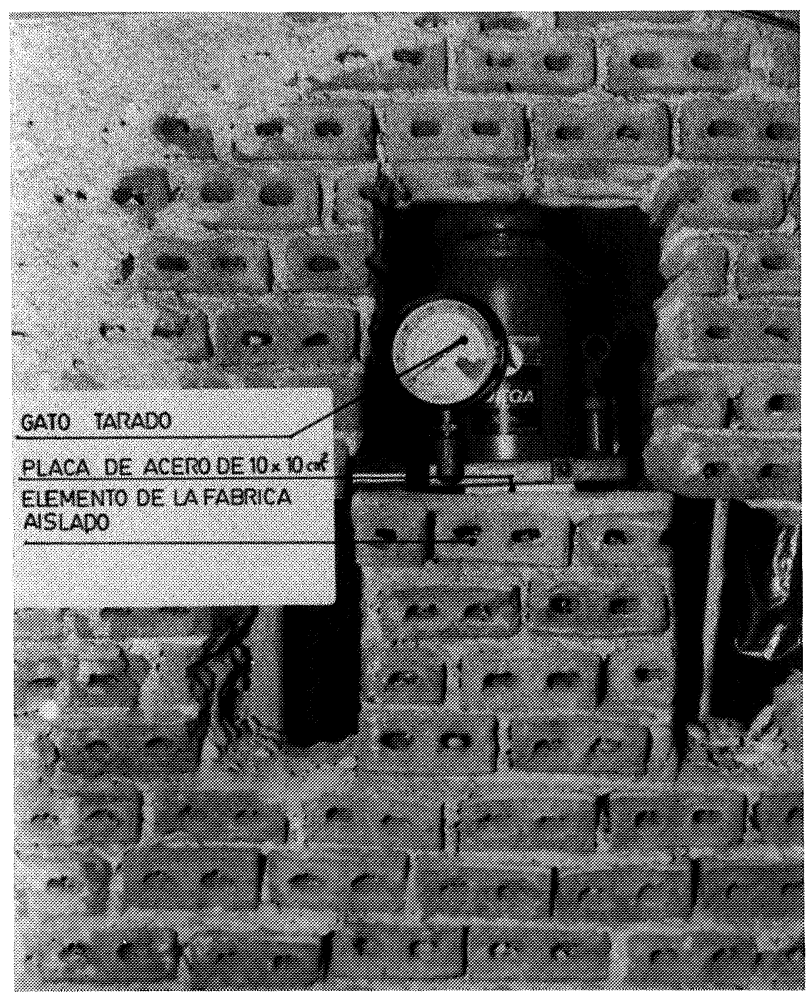

Fig. 6. - Determinación de resistencia de una fábrica de ladrillo "in situ". han ensayado varias probetas (Fig. 5) de ladrillo macizo y hueco cuya rotura se ha efectuado a través de una placa de acero de superficie $S_{1}$, siendo $S_{2}$ la de la probeta. Variando $S_{1}$ se ha obtenido una correlación entre la fatiga última $\mathrm{N}_{\mathrm{u}}$ :

$$
\sigma_{\mathrm{cr}}=\frac{\mathrm{N}_{\mathrm{u}}}{\mathrm{S}_{1}}
$$

y la relación $S_{2}: S_{1}$; introduciendo luego el factor de esbeltez $\lambda$, se obtiene el gráfico de la figura 4 .

La forma de utilización en obra queda aclarada en la figura 6. Es preciso aislar un elemento de este muro con ayuda de una sierra o punzón y todo ello con sumo cuidado, para que la muestra no quede deteriorada en sus flancos. El buen sentido del proyectista unirá los datos así obtenidos a los que le dicten las tablas estimativas de resistencia para decidir los valores a emplear finalmente, con la suficiente prudencia.

\section{RASGADO DE HUECOS EN MUROS CIEGOS}

La figura 7 muestra un rasgado en muro ciego. Trataremos un caso concreto con valores numéricos ya desde el principio, lo cual resultará más asimilable al lector y no quita, como se verá, generalidad y rigor a la exposición. Lo contrario - tratar el proble-

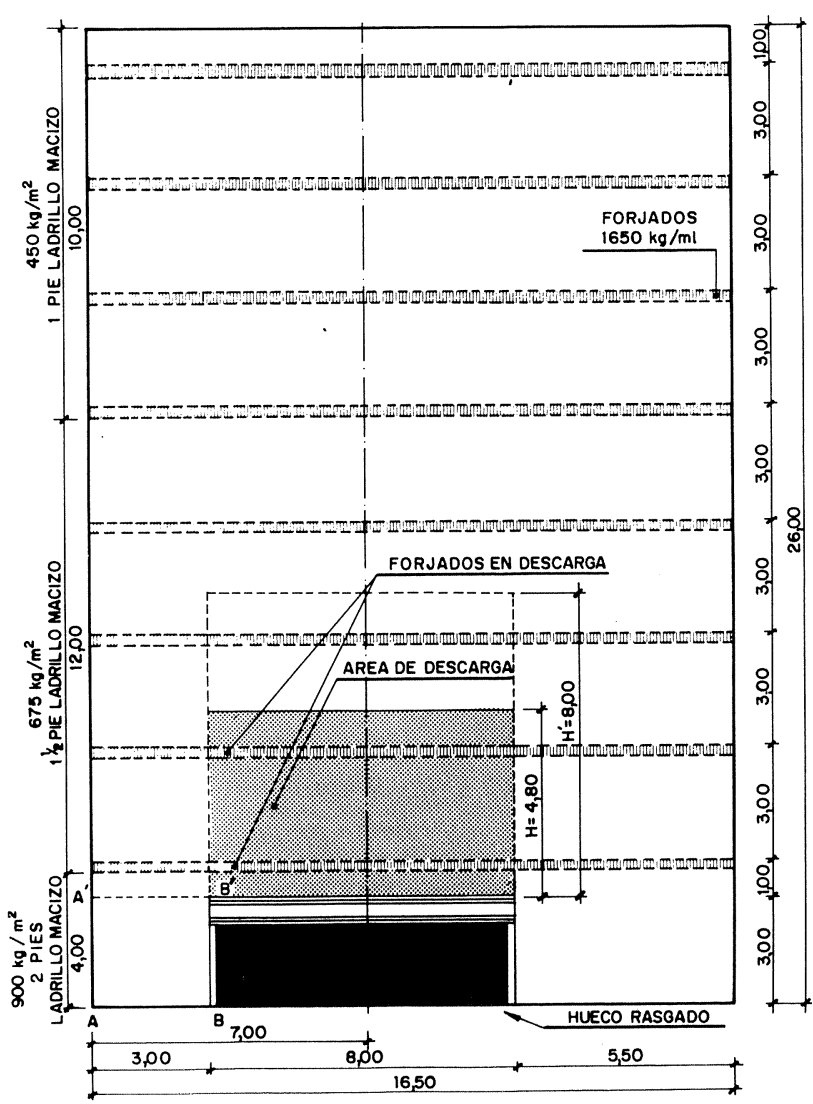

Fig. 7.-Rasgado de un muro ciego. Cargas de cálculo. (Son 3 los forjados en lugar de los 2 indicados). 
ma con parámetros literales- conduciría a enojosas foŕmulas inútiles, por otro lado, en este caso.

Arco de descarga: Según MV-201-1972 el área gravitante es un rectángulo de dimensiones $\mathrm{L}$ y $\mathrm{H}=0$, $6 \mathrm{~L}=4,80$, además de los forjados comprendidos en la altura $\mathrm{H}=\mathrm{L}$.

$$
\begin{aligned}
& \text { Cargas: Muros: } \quad 8,00 \cdot 1,00 \cdot 900=7.200 \mathrm{~kg} \\
& 8,00 \cdot 3,80 \cdot 675=20.520 \mathrm{~kg} \\
& \text { Forjados: } 3 \quad \cdot 8,00 \cdot 1.650=39.600 \mathrm{~kg} \\
& \text { Total.......67.320 kg }
\end{aligned}
$$

Preflexión o puesta en carga del dintel $P_{1}=67.320 \mathrm{~kg}$ (supuesto solamente el problema de la fisuración del arco)

\section{Tensiones en la pilastra AB:}

Carga unitaria en $A B$ después de la preflexión $P_{1}$ :

$$
\begin{aligned}
& \text { Muros: } \quad 7[(3 \cdot 3,00+1) \cdot 450+ \\
& +3 \cdot 4,00 \cdot 675+1,00 \text {. } \\
& \text {-900] }+3,00 \cdot 3,00 \text {. } \\
& \cdot 900=102.600 \mathrm{~kg} \\
& \text { Forjados: } 8 \cdot 7,00 \cdot 1.650 \quad=\underline{92.400 \mathrm{~kg}} \\
& \text { A deducir: } \frac{\mathrm{P}_{1}}{2} \quad=\quad 33.660 \mathrm{~kg} \\
& \text { Total. ......... }=161.340 \mathrm{~kg} \\
& \sigma=\frac{161.340}{300 \cdot 50}=10,75 \mathrm{~kg} / \mathrm{cm}^{2}
\end{aligned}
$$

Esta fábrica había dado en el ensayo de carga concentrada: $S_{2}=50 \cdot 50=2.500 \mathrm{~cm}^{2} ; S_{1}=15 \cdot 15=$ $=225 \mathrm{~cm}^{2}$ :

$$
\frac{\mathrm{S}_{2}}{\mathrm{~S}_{1}}=11 \quad \sigma_{\mathrm{cr}}=\frac{35.000}{225}=155 \mathrm{~kg} / \mathrm{cm}^{2}
$$

Llevando estos valores al gráfico de la figura 4, tendremos:

$$
\begin{aligned}
& \lambda=\frac{300}{50}=6 \quad \sigma_{\mathrm{ad}_{\lambda=6}}=\sigma_{\mathrm{cr}}: 20=155: 20= \\
& =7,77 \mathrm{~kg} / \mathrm{cm}^{2}
\end{aligned}
$$

Vemos que la pilastra $A B$ soporta una tensión $10,75>7,77$ por lo que es preciso descargarla en la cuantía de:

$$
(10,75-7,77) \cdot 300 \cdot 50=44.700 \mathrm{~kg}
$$

mediante una preflexión adicional $P_{2}$.

\section{Actuación y eficacia de la preflexión $P_{\mathbf{2}}$}

Esta preflexión, que se sitúa junto al punto $B^{\prime}$ en un tramo de dintel tan reducido como se pueda a fin de que repercuta lo más posible en la zona $A^{\prime} B^{\prime}$, no se aplica en su totalidad al fin perseguido, pues sufre una cierta dispersión hacia otras zonas.

En la figura 8 , suponemos que los flujos de líneas de fuerza que llegan a $A B$ y los que salen de $A C\left(P_{2}\right)$ lo hacen con una dispersión $\alpha$.

Area de influencia sobre $A B=A^{\prime} A B B^{\prime}=S_{2}+S_{3}$ Area de influencia de $A C=C^{\prime} C A A^{\prime \prime}=S_{1}+S_{2}=\left(P_{2}\right)$ Zona desaprovechada de la acción de $P_{2}: S_{1}$.

Grado de aprovechamiento o influencia de $P_{2}$ :

$$
\delta=100 \frac{\mathrm{S}_{2}}{\mathrm{~S}_{2}+\mathrm{S}_{1}}=100 \cdot \operatorname{tg} \alpha:\left(\frac{\mathrm{a}}{\mathrm{H}}+\operatorname{tg} \alpha\right)
$$

Tomando $\alpha \simeq 30^{\circ}$ se tiene:

$$
\delta=60:\left(\frac{\mathrm{a}}{\mathrm{H}}+0,60\right)<100(\%)
$$

Por tanto, para obtener un valor eficaz $\left(P_{2}\right)$ había que introducir una preflexión:

$$
P_{2}=\frac{P_{2}}{\delta} \cdot 100=P_{2} \frac{\frac{a}{H}+0,60}{0,60}
$$$$
\text { Con } \mathrm{a}=0,60 \mathrm{~m} \text { y } \mathrm{H}=21 \mathrm{~m} \quad \mathrm{P}_{2}=1,16\left(\mathrm{P}_{2}\right)=1,16 \text {. }
$$$$
\cdot 44.700=51.852 \mathrm{~kg}
$$

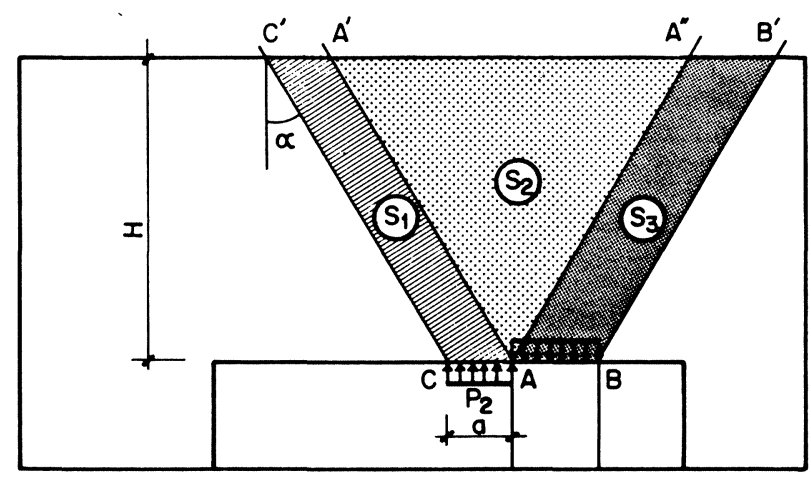

Fig. 8. -Grado de eficacia de la preflexión $P_{2}$ sobre $A B$.

\section{Cargas de contacto dintel-fábrica}

En la zona de aplicación de $P_{2}$ (en la que coincide también $P_{1}$ ) (ver Fig. 9, arriba, zona 1), se tiene:

Carga dimanante de $P_{1}: 67.320 \cdot \frac{2,00}{8,00}=16.830$ 


\section{ESQUEMA DE CARGAS}

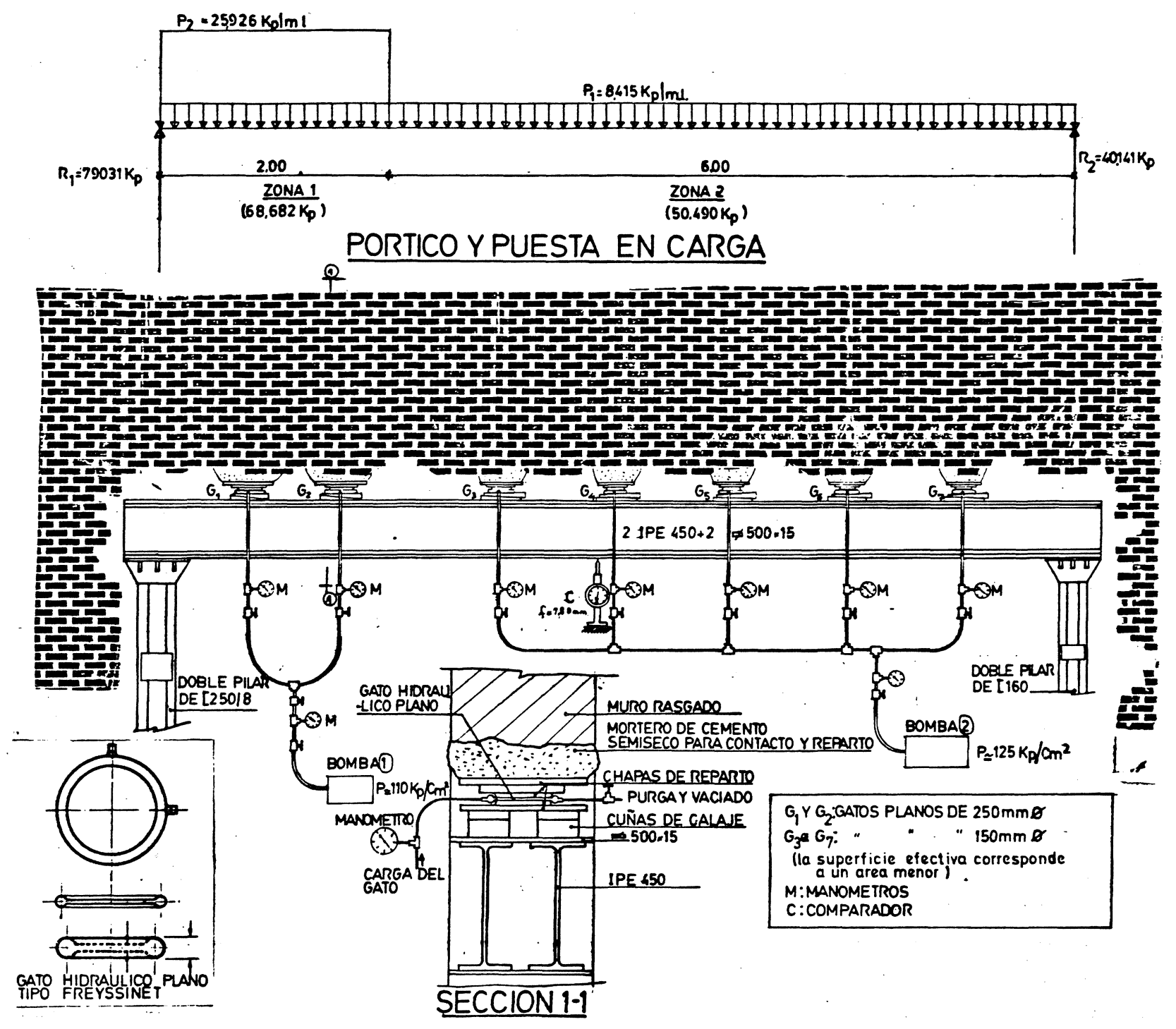

Fig. 9.-Solución de dintel para el caso de la figura 7 con indicación de cargas, perfiles metálicos y sistema de preflexión.

Carga dimanante de $\dot{P}_{2}$ :

$$
\text { Total: } \quad 68.682
$$

$\sigma=\frac{68.682}{200 \cdot 50}=6,86 \mathrm{~kg} / \mathrm{cm}^{2}<7,77$ (admisible)

\section{Cálculo del pórtico metálico}

Con las cargas de la figura 9, obtenemos:

$M_{\text {máx }}=95.476 \mathrm{~m} / \mathrm{kp}$ y reacciones $R_{1}=79.031 \mathrm{kp} \mathrm{y}$ $\mathrm{R}_{2}=40.141 \mathrm{kp}$.

Dintel: dos IPE 450 con dos platabandas de $500 \cdot 15$ que dan:

$$
I=148.575 \mathrm{~cm}^{4}, W=6.190 \mathrm{~cm}^{3}
$$

Flecha máxima: $f_{\text {máx }}=7,80 \mathrm{~mm}$ para $\chi=3,85 \mathrm{~m}$.

Pilares: se indican en la figura y son de sección distinta como corresponde a distintas cargas.

\section{Puesta en carga}

En la figura 9 se detalla la operación de preflexión. Se utilizan gatos hidráulicos planos (tomados del catálogo de Freyssinet), que pueden quedar al final perdidos, previo relleno de los mismos con resina o pasta de cemento. En caso contrario deberá acuñarse junto a los gatos, para sustituir a éstos, extrayéndolos.

Se colocará un comparador para detectar la flecha del dintel bajo las cargas $P_{1}$ y $P_{2}$, que en este caso 
-omito el cálculo- es de $\mathrm{f}=7,80 \mathrm{~mm}$ a $\chi=3,85 \mathrm{~m}$. Este valor es el que realmente garantiza la correcta puesta en carga y no la presión calculada en los gatos.

El proceso de preflexión ha de ser lento, elevando paulatinamente las cargas y permitiendo que se estabilicen durante un tiempo prudencial, pues tienden a bajar. Previamente habremos comprobado la presión de contacto gato-fábrica, utilizando palastros para llegar a la superficie necesaria y una capa de pasta de cemento que pueda adaptarse a las irregularidades del rasgado. Téngase muy presente que las cargas $P_{1}$ y $P_{2}$ se han calculado a partir de unos pesos propios supuestos. Por ello habrá que observar el edificio, en el proceso de carga, deteniendo ésta en un valor más bajo si se observasen fisuras por levantamiento del mismo o incluso simples ruidos extraños, producidos normalmente por quiebros interiores. Estaríamos creando un asiento negativo.

Obviamente esta operación debe confiarse a alguien especializado que no solamente aportará los elementos precisos, sino su experiencia.

\section{RASGADO EN MUROS DOTADOS DE HUECOS}

Cuando el muro a rasgar no es ciego, cual es el caso corriente de fachadas, por la presencia de ventanas y balcones o de crujías interiores por los huecos de paso, el arco de descarga no se limita al área de la figura 7 , sino que abarca prácticamente la totalidad de la zona comprendida entre el dintel y la coronación del muro (Fig. 10).

Pueden presentarse casos intermedios de fachadas con escasa proporción de huecos, compuestos además por material muy eficaz a la tracción, en las que cabría estudiar el arco real de descarga. Esto, que conduciría a una cierta economía en la operación, es complicado, además de algo incierto, por lo que eludo su estudio aquí (*).

Dentro de las cargas a considerar existe un sumando variable: la sobrecarga de uso. Esta no se aplica totalmente casi nunca y además su valor es variable con el tiempo, de suerte que si efectuamos una preflexión a sobrecarga $100 \%$ de cálculo, tendremos un cierto desequilibrio dintel-área gravitante, en sentido ascendente, pudiéndose llegar a un efecto de "asiento negativo" por exceso de preflexión. Forzoso es que exista siempre un cierto desequilibrio, el cual debe reducirse al mínimo y para ello tomaremos como factor sobrecarga la mitad de la de cálculo.

(*) El procedimiento de cálculo se expone en el apartado «igrietas residuales? que allí se aplica al $50 \%$ de la sobrecarga de uso y aquí tendría que aplicarse a la carga total.

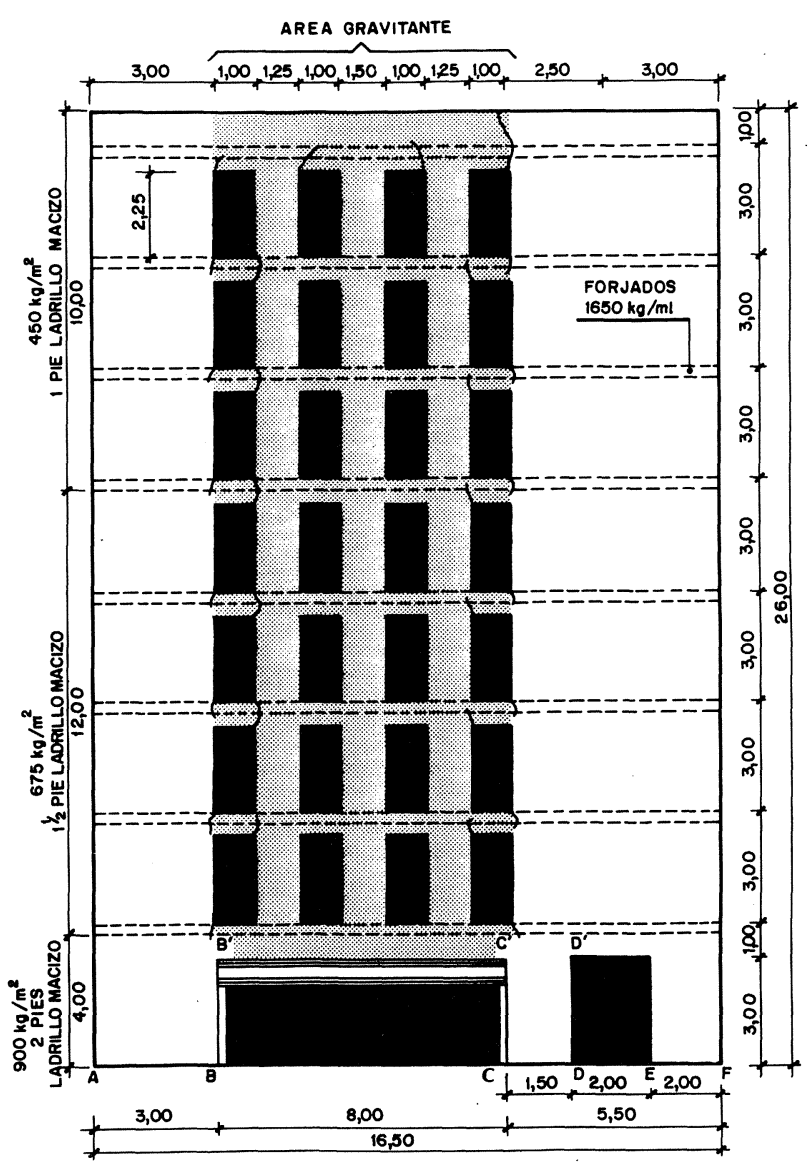

Fig. 10.-Rasgado en un muro dotado de huecos. Grietas típicas de una ejecución imperfecta, sin puesta en carga.

En la figura 10 tendremos:

Cargas de muro: $(8,00 \cdot 10,00-12$.

$$
\cdot 2,25 \cdot 1,00) \cdot 450=23.850
$$

(con descuento

de huecos) \& $(8,00 \cdot 12,00-16$.

$$
\begin{aligned}
& 2,25 \cdot 1,00) \cdot 675=40.500 \\
& 8,00 \cdot 1,00 \cdot 900=7.200
\end{aligned}
$$

Forjados: $8 \cdot 8,00 \cdot 1.650=105.600$

Deducir, $50 \%$ sobrecarga

de uso:

$$
\frac{50}{100} \cdot 0,30 \cdot 105.600-15.840=\frac{89.760}{161.310}
$$

Preflexión: $P_{1}=161.310 \mathrm{kp}$

\section{Situación de la pilastra CD:}

Cargas en $C^{\prime} D^{\prime}$ (área comprendida entre $C^{\prime} C^{\prime \prime}$ y el eje vertical de $D E)$ :

Muro: $2,50(10,00 \cdot 450+12,00 \cdot 675+$

$+1 \cdot 900)$
Forjado (sin disminución de sobrecarga):

$$
8 \cdot 2,50 \cdot 1.650=33.000
$$


$\sigma=\frac{66.750}{150 \cdot 50}=8,90 \mathrm{kp} / \mathrm{cm}^{2}>7,77\left(=\sigma_{\mathrm{ad}_{\lambda}=6}\right)$

luego hay que reducir el valor de $\sigma$. Hay dos soluciones:

\section{Solución 1: Preflexión adicional}

$$
\mathbf{P}_{2}=(8,90-7,77) \cdot 150 \cdot 50=8.475 \mathrm{kp} .
$$

Adoptando un coeficiente de eficacia $\delta=0,80$ :

$$
P_{2}=\frac{8.475}{0,80}=10.593 \mathrm{kp}
$$

El conjunto $P_{2}+P_{1}$ supera las cargas existentes sobre el dintel $B^{\prime} C^{\prime}$ por lo que hay peligro de asientos negativos. Esta solución es válida para pequeños valores de $P_{2}$, estudiando bien su repercusión en el resto del muro. Es preferible, siempre que se pueda, la solución 2 .

\section{Solución 2: Refuerzo de la pilastra}

Antes de proceder al rasgado se refuerza la pilastra (Fig. 11). En este caso hemos optado por el zunchado de la pieza mediante una camisa de hormigón armado (Fig. 12). Comprobamos la aptitud del refuerzo:

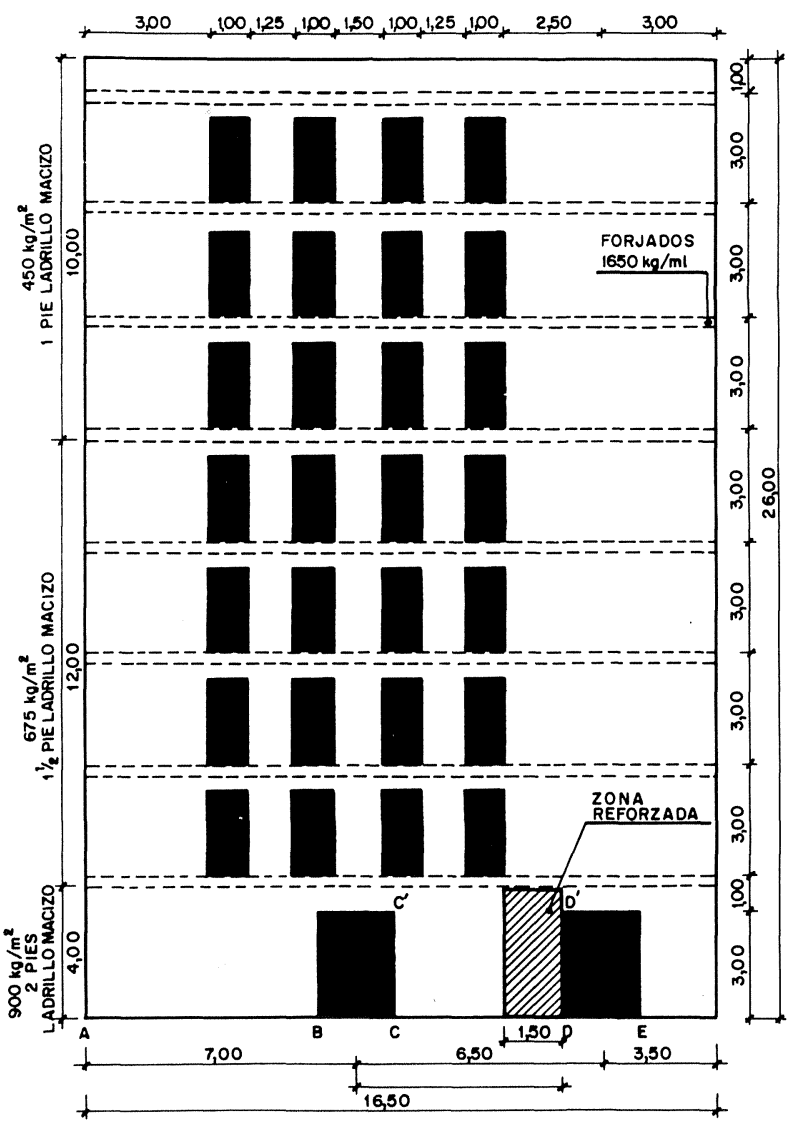

Fig. 11.-Solución de refuerzo de pilastra sobrecargada antes de proceder al rasgado.
Tensión inicial en $\mathrm{C}^{\prime} \mathrm{D}^{\prime}$ antes del rasgado (Fig. 11):

$$
\begin{array}{ll}
\text { Muros: } \begin{array}{l}
6,50(10,00 \cdot 450+12,00 \\
\cdot 675+1,00 \cdot 900)
\end{array}=87.750 \\
\text { Huecos: } \begin{array}{l}
-[6,00 \cdot 1,00 \cdot 2,25 \cdot 450+ \\
+8 \cdot 1,00 \cdot 2,25 \cdot 675]
\end{array}=-18.225 \\
\text { Forjados: } 8 \cdot 6,50 \cdot 1.650= & \frac{85.800}{155.325} \\
\sigma_{0}=\frac{155.325}{450 \cdot 50}=6,90 \mathrm{kp} / \mathrm{cm}^{2}
\end{array}
$$

\begin{tabular}{|c|c|c|c|c|c|}
\hline Material & $\begin{array}{l}\text { Módulo } \\
\left(\mathbf{k p} / \mathrm{cm}^{2}\right)\end{array}$ & $\begin{array}{c}\text { Sección } \\
\mathrm{cm}^{2}\end{array}$ & $\begin{array}{l}\text { Relación } \\
\text { de } \\
\text { módulos }\end{array}$ & $\begin{array}{c}\text { Sección } \\
\text { equival. } \\
\left(\mathrm{cm}^{2}\right)\end{array}$ & $\begin{array}{l}\text { Distribu- } \\
\text { ción } \\
(\%)\end{array}$ \\
\hline 1 & $\begin{array}{r}35 \cdot 10^{3} \\
8 \cdot 10^{5}\end{array}$ & $\begin{array}{l}7.000 \\
3.380\end{array}$ & $\begin{array}{c}1 \\
22,85\end{array}$ & $\begin{array}{r}7.000 \\
77.257\end{array}$ & $\begin{array}{r}8,01 \\
89,21\end{array}$ \\
\hline & $2,1 \cdot 10^{6}$ & 40,19 & 60 & 2.411 & 1,78 \\
\hline
\end{tabular}

Pilastra reforzada: A partir del valor $\sigma_{0}$ se producen unos aumentos de tensión que se reparten entre los materiales (ladrillo, acero y hormigón) proporcionalmente a su sección y a su módulo $\mathrm{E}$ de elasticidad.

Reducimos las secciones a una equivalente de ladrillo:

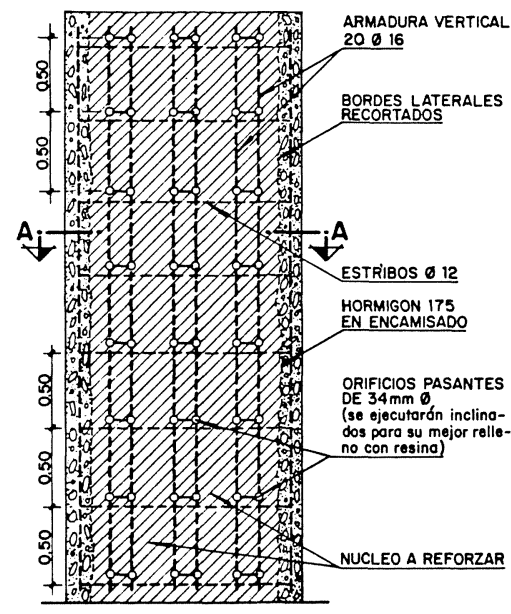

ALZADO

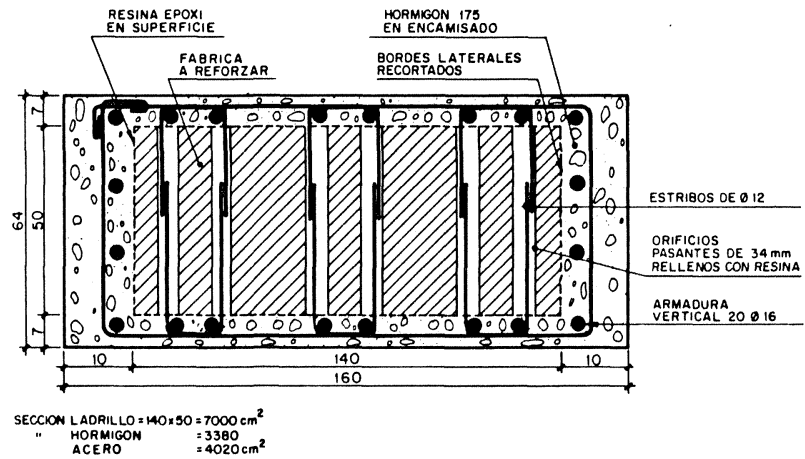

Fig. 12.-Sistema de refuerzo de pilastra por zunchado con una camisa de hormigón armado solidario con la fábrica. 
Carga total final en pilastra $=66.750$ Carga correspondiente al estado inicial:

$$
140 \cdot 50 \cdot 6,90
$$

Aumento por el rasgado

$=18.450$

$$
\Delta \sigma=\frac{18.450}{86.668}=0,21 \mathrm{kp} / \mathrm{cm}^{2}
$$

Tensión final:

$$
\sigma=\sigma_{0}+\Delta \sigma=6,90+0,21=7,11 \mathrm{kp} / \mathrm{cm}^{2}
$$

Tensión admisible con:

$$
\begin{gathered}
\lambda=\frac{300}{64}=4,68 \text { (Fig. 4) } \\
\sigma_{\mathrm{ad}_{\lambda=4,68}}=\frac{\sigma_{\mathrm{cr}}}{\mathrm{k}}=\frac{155}{17}=9,11 \mathrm{kp} / \mathrm{cm}^{2}
\end{gathered}
$$

$\left(\sigma_{\mathrm{cr}}=155 \mathrm{kp} / \mathrm{cm}^{2}\right.$ con $S_{2}=S_{1}=11$, recuérdese).

\section{$7,11<9,11$, luego el refuerzo es válido.}

Podríamos haber tenido en cuenta, además, el efecto de zunchado. A veces no es viable reforzar la pilastra por sus cuatro caras, ya sea por inaccesibilidad de alguna, ya sea porque, v.g. una corresponde a fachada y no se admite su regruesado. Podemos, en tal caso, reforzar por dentro, para lo cual reduciremos la esbeltez. Con $\lambda=4,75$ tenemos $\sigma_{\mathrm{ad}}=8,90 \mathrm{kp} / \mathrm{cm}^{2}$. Bastará adosar interiormente unos perfiles metálicos gruesos que hagan de rigidizadores, debidamente conectados a la pilastra para lograr en ésta una mayor inercia en su sección horizontal y por tanto la reducción de $\lambda$. Omitimos el cálculo correspondiente.

¿Grietas residuales?: Ya hemos visto que existe un término variable - el $50 \%$ de la sobrecarga accidental- que no se compensa con la preflexión del dintel. Su variabilidad, a lo largo de la vida del edificio, deberá ser absorbida por la propia rigidez del entramado del muro. Si el muro no tiene rigidez por ser la proporción de huecos muy grande o la calidad del material muy baja, no podrá absorberlo y se producirán unas grietas inevitables y cambiantes con la sobrecarga.

A continuación se expone un camino de cálculo para averiguar si se producirán grietas residuales.

El paño existente sobre el dintel (Fig. 13) se conecta con el resto del muro a través de los elementos rayados, cada uno de los cuales absorbe un cortante, $S_{n}$, proporcional a la rigidez a flexión del elemento $S_{n}$, proporcional a su vez al valor $I=b h^{3}: 12$ ya que $l$ es en todos igual.

De lo dicho se desprende, en la página siguiente, un cuadro de cálculo, en el cual se trata de averiguar
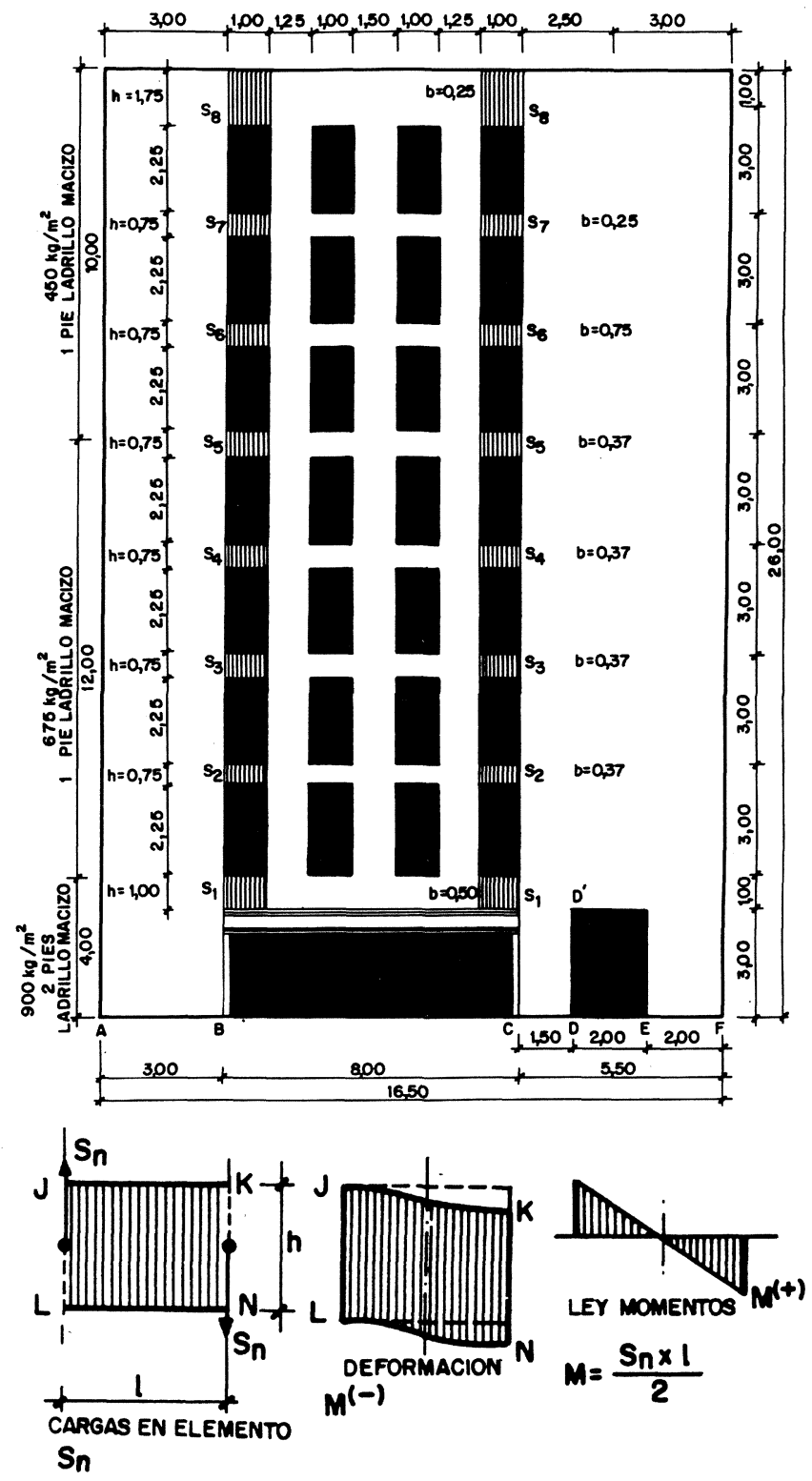

Fig. 13.-Estudio de la rigidez propia del muro.

dónde se da la máxima $\sigma$ de flexión y $\tau$ de cortante, comprobando si estos valores resultan aceptables.

Como valor aceptable para $\sigma$ y $\tau$ puede tomarse el $10 \%$ del de rotura a compresión:

$\sigma_{\mathrm{ad}} \simeq \tau_{\mathrm{ad}}=0,10 \cdot \sigma_{\mathrm{cad}}$

$\sigma_{\mathrm{c}_{\mathrm{ad}}}=\frac{155}{4,5}=34 \mathrm{kp} / \mathrm{cm}^{2}$ (de Fig. 4)

$\sigma_{\mathrm{ad}}=\tau_{\mathrm{ad}}=3,40 \mathrm{kp} / \mathrm{cm}^{2}>0,90>0,88$

valores máximos en el entramado al ser sometido a la carga variable máxima de $15.840 \mathrm{kp}$, por lo que no habrá grietas residuales. 


\section{CALCULO DEL ESTADO DE TENSIONES EN LA FABRICA (ZONAS RAYADAS) POR EFECTO DEL 50 \% DE LA SOBRECARGA}

\begin{tabular}{|c|c|c|c|c|c|c|}
\hline$A$ & Elemento n. $^{\circ}$ & $S_{1}$ & $\mathrm{~S}_{2}$ a $\mathrm{S}_{5}$ & $S_{6}$ a $S_{7}$ & $S_{8}$ & Total \\
\hline B & N. ${ }^{\circ}$ unidades & 2 & 8 & 4 & 2 & 16 \\
\hline $\mathrm{C}$ & $\mathrm{b}(\mathrm{cm})$ & 50 & 37,5 & 25 & 25 & \\
\hline $\mathrm{D}$ & $\mathrm{h}(\mathrm{cm})$ & 100 & 75 & 75 & 175 & \\
\hline$E$ & $\mathrm{I}(\mathrm{cm})$ & 100 & 100 & 100 & 100 & \\
\hline $\mathrm{F}$ & $\mathrm{b} \cdot \mathrm{h}^{3}\left(\mathrm{~cm}^{4}\right) \cdot 10^{-7}$ & 5 & 1,6 & 1,05 & 13,39 & \\
\hline G & n. ${ }^{o}$ uds $\cdot b h^{3} \cdot 10^{-7}$ & 10 & 12,80 & 4,20 & 26,78 & 53,78 \\
\hline $\mathrm{H}$ & $\begin{array}{l}\text { Distribución en \% línea } \\
\text { anterior }\end{array}$ & 18,60 & 23,80 & 7,80 & 49,80 & 100,00 \\
\hline 1 & $\begin{array}{l}\text { Valores de } S= \\
=\frac{15.840 \cdot(\mathrm{H})}{(\mathrm{B}) \cdot 100}(\mathrm{kp})\end{array}$ & 1.473 & 471,24 & 308,88 & 3.944 & \\
\hline$J$ & $\begin{array}{l}M=\frac{S \cdot 1}{2}= \\
=\frac{S \cdot 100}{2}(\mathrm{kp} / \mathrm{cm})\end{array}$ & 73.650 & 23.550 & 15.450 & 197.200 & \\
\hline K & $w=\frac{b \cdot h^{2}}{6}\left(\mathrm{~cm}^{3}\right)$ & $8,30 \cdot 10^{4}$ & $3,51 \cdot 10^{4}$ & $2,51 \cdot 10^{4}$ & $22,33 \cdot 10^{4}$ & \\
\hline $\mathrm{L}$ & $\sigma=\frac{M}{W}=\frac{(J)}{(K)}\left(\mathrm{kp} / \mathrm{cm}^{2}\right)$ & 0,88 & 0,67 & 0,61 & 0,88 & \\
\hline$M \tau$ & $\tau=\frac{S_{n}}{b \cdot 0,7 \cdot h}\left(\mathrm{kp} / \mathrm{cm}^{2}\right)$ & 0,42 & 0,17 & 0,16 & 0,90 & \\
\hline
\end{tabular}

\section{LIMITES DEL VALOR DE LA PREFLEXION, EN FUNCION DE LA RIGIDEZ DE LA FABRICA A RASGAR}

El estudio de grietas residuales realizado sobre la figura 13 sirve también para obtener los límites máximo y mínimo de la preflexión, compatibles con la resistencia de la fábrica.

El valor máximo será aquél cuya resultante hacia arriba supuesta una sobrecarga de uso nula, produzca los valores $\sigma_{\mathrm{ad}}$ y $\tau_{\mathrm{ad}}$. El mínimo será el que, con la sobrecarga de uso máxima (la nominal), dé lugar a unas acciones hacia abajo de valor $\sigma_{\mathrm{ad}}$ y $\tau_{\mathrm{ad}}$. Resultante que produce $\sigma_{\mathrm{ad}}$ y $\tau_{\mathrm{ad}}$ (Fig. 13 y cálculos siguientes):

$$
R=\frac{3,40}{0,90} \cdot 15.840=59.840 \mathrm{kp}
$$

Máximo valor de $P_{1}$ :

$\left(P_{1}\right)_{\text {máx }}-($ peso de la fábrica sin sobrecarga $)=$ $=59.840 \mathrm{kp}$

$\left(P_{1}\right)_{\text {máx }}=59.840+161.310-15.840=205.310 \mathrm{kp}$

Mínimo valor de $P_{1}$ :

(Peso de la fábrica con sobrecarga nominal) $-\left(P_{1}\right)_{\min }=59.840 \mathrm{kp}$
$\left(P_{1}\right)_{\min }=(161.310+15.840)-59.840=$ $=117.310 \mathrm{kp}$

Por tanto:

$\left(P_{1}\right)_{\min }=117.310<P_{1}=161.310<\left(P_{1}\right)_{\min }=$ $=205.310 \mathrm{kp}$

Margen de tolerancia:

$161.310-117.310=44.000 \mathrm{kp}$ en menos.

$205.310-161.310=44.000 \mathrm{kp}$ en más.

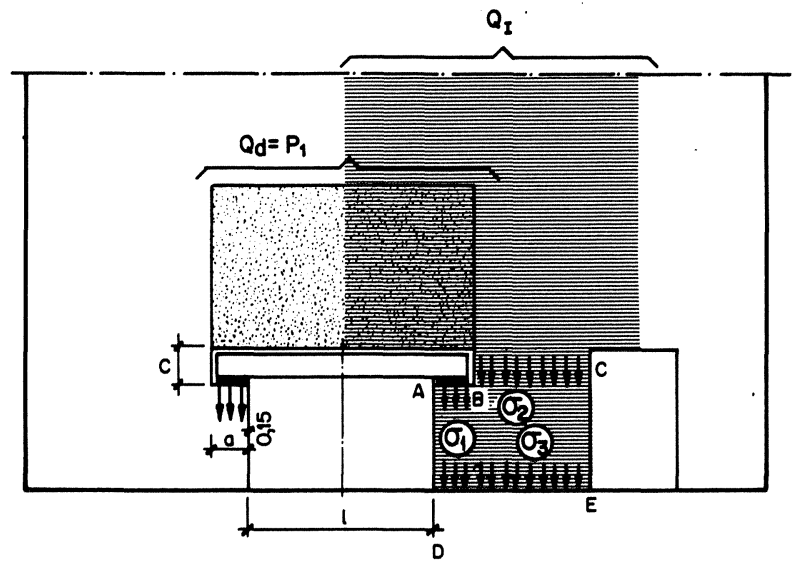

Fig. 14.-Cargaderos apoyados en fábrica. Cargas de cálculo. 
El margen es, pues, de $\pm 44.000 \mathrm{kp}$ equivalente a un $\pm 27 \%$ aproximadamente del valor adoptado, lo cual permite acometer la operación con cierta tranquilidad pese a lo inexacto de la estimación de pesos propios de la fábrica, tranquilidad que no debe disminuir nunca las precauciones recomendadas en el proceso de puesta en carga.

\section{CARGADEROS APOYADOS EN LA PROPIA FABRICA. SUPRESION DE PILARES}

Apoyar el dintel metálico en unas esperas realizadas en la propia fábrica es práctica harto frecuente que no presenta inconvenientes para luces pequeñas, siendo desaconsejable cuando éstas son importantes. Según la magnitud del cajeado a (Fig. 14), se dividen los cargaderos en apoyados ( $c>a$ ) y empotrados $(c<a)$. Omito el tratamiento de estos últimos ya que un empotramiento real y efectivo exige un valor a grande y una ejecución muy cuidada que presumiblemente no se dará; la fábrica quedará muy castigada, sin contrapartidas apreciables.

Cargaderos apoyados: Consideraremos vacío, a efectos de transmisión de cargas, el vano de entrega a, por mucho cuidado que pongamos en retacarlo con mortero una vez colocado el dintel.

No procede en estos casos utilizar la segunda preflexión $P_{2}$, encaminada a proteger la pilastra $D E$, dado que si bien ello conduciría a disminuir $\sigma_{2}$, no alteraría $\sigma_{3}$ porque en la base $D E$ se recibiría nuevamente $\mathrm{P}_{2}$ procedente de la reacción de apoyo en $\mathrm{AB}$.

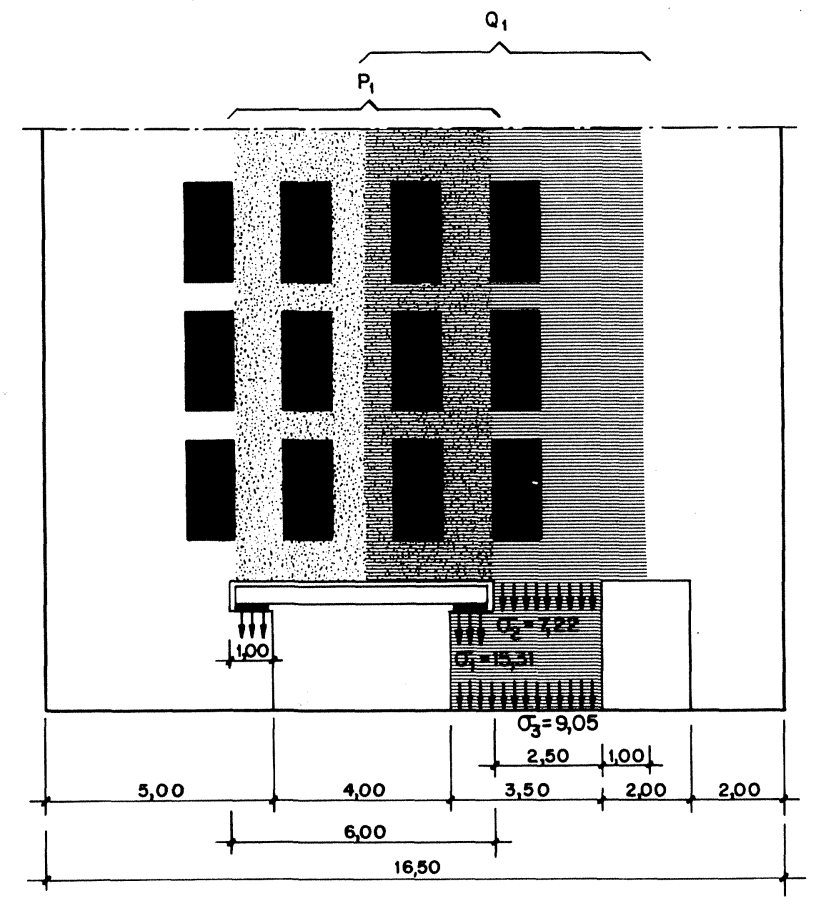

Fig. 15.-Cargadero apoyado en íábrica. Aplicación práctica.
De aquí que si el rasgado implica un $\sigma_{2}$ o un $\sigma_{3}$ excesivo, la única solución sea el refuerzo de la pilastra, o el empleo de pilares metálicos - como veremos en el ejemplo siguiente- renunciando al apoyo en fábrica.

La longitud de apoyo es a-15 cm ya que el borde no se utiliza.

$$
\begin{gathered}
\sigma_{1}=\frac{\mathrm{P}_{1}}{2(\mathrm{a}-0,15) \mathrm{b}} \leq \sigma_{\mathrm{ad}(\lambda=0)} \\
\sigma_{3}=\frac{\mathrm{Q}_{1}}{\overline{\mathrm{DE}}}<\sigma_{\mathrm{ad}(\lambda)} \\
y \sigma_{2}=\frac{\mathrm{Q}_{1}-\frac{\mathrm{P}_{1}}{2}}{\overline{\mathrm{BC}} \cdot \mathrm{b}} \leq \sigma_{\mathrm{ad}(\lambda)}
\end{gathered}
$$

Aplicación a la fachada de casos anteriores para $\mathrm{I}=4,00 \mathrm{~m}$ (Fig. 15)

$P_{1}(10,00 \cdot 6,00-6 \cdot 1,00 \cdot 2,25)$.

$$
\begin{array}{lrl}
\cdot 450 & = & 20.925 \mathrm{~kg} \\
(12,00 \cdot 6,00-8 \cdot 1,00 \cdot 2,25) & \cdot \\
\cdot 675 & =36.450 \mathrm{~kg} \\
1,00 \cdot 6,00 \cdot 900 & =5.400 \mathrm{~kg} \\
8 \cdot 6,00 \cdot 1.650 & =79.200 \mathrm{~kg} \\
50 \% \text { sobrecarga: } & & =-11.880 \mathrm{~kg} \\
-0,5 \cdot 0,30 \cdot 79.200 & &
\end{array}
$$

$$
\begin{gathered}
\sigma_{1}=\frac{130.095}{2(100-15) \cdot 50}=15,31 \mathrm{kp} / \mathrm{cm}^{2} \\
\text { c.s. }=\frac{34,00}{15,31}=2,22 \\
Q_{1}-\frac{P_{1}}{2}:
\end{gathered}
$$

$(10,00 \cdot 3,50-3 \cdot 1,00 \cdot 2,25) \cdot$

$$
\cdot 450
$$$$
(12,00 \cdot 3,50-4 \cdot 1,00 \cdot 2,25) \text {. }
$$

$\cdot 675$

$1,00 \cdot 3,50 \cdot 900$

$8 \cdot 3,50 \cdot 1.650$ $=22.275 \mathrm{~kg}$ $=3.150 \mathrm{~kg}$ $=46.200 \mathrm{~kg}$

Hay que añadir el $50 \%$ de la sobrecarga deducida en $\mathrm{P}_{1}$ que puede aparecer y transmitirse por la propia fábrica hasta $\overline{\mathrm{BC}}$.

$$
\frac{1}{2} \cdot 11.880
$$




$$
Q_{1}-\frac{P_{1}}{2} \quad=90.277 \mathrm{~kg}
$$

$\sigma_{2}=\frac{90.277}{250 \cdot 50}=7,22<7,77 \mathrm{kp} / \mathrm{cm}^{2}$ (admisible)

$$
Q_{1}=90.277+\frac{136.156}{2}=158.355 \mathrm{~kg} \quad Q_{1}-\frac{P_{1}}{2} \text { : }
$$

$\sigma_{3}=\frac{158.355}{350 \cdot 50}=9,05>7,77 \mathrm{kp} / \mathrm{cm}^{2}$ (inadmisible)

Por tanto habría que recurrir a reforzar la pilastra o bien apoyar el dintel en pilares metálicos, con cuya solución las cifras hubieran sido:

$P_{1}$ (ancho de 4,00 m):

$$
\begin{aligned}
& (10,00 \cdot 4,00-6 \cdot 1,00 \cdot \\
& \cdot 2,25) \cdot 450 \\
& (12,00 \cdot 4,00-8 \cdot 1,00 \cdot \\
& \cdot 2,25) \cdot 675 \\
& 1,00 \cdot 4,00 \cdot 900 \\
& 8 \cdot 4,00 \cdot 1.650 \\
& -0,5 \cdot 0,3 \cdot 52.800
\end{aligned}
$$$$
=11.925 \mathrm{~kg}
$$$$
=20.250 \mathrm{~kg}
$$$$
=3.600 \mathrm{~kg}
$$$$
=52.800 \mathrm{~kg}
$$$$
=-7.920 \mathrm{~kg}
$$$$
\mathrm{P}_{1}=80.655 \mathrm{~kg}
$$

$P_{1}$ representa la carga en pilares.

$$
\begin{aligned}
& (10,00 \cdot 4,50-3 \cdot 1,00 \cdot 2,25) \text {. } \\
& \cdot 450=17.212 \mathrm{~kg} \\
& (12,00 \cdot 4,50-4 \cdot 1,00 \cdot 2,25) \text {. } \\
& \text { - } 675 \\
& 1,00 \cdot 3,50 \cdot 900 \\
& 8 \cdot 4,50 \cdot 1.650 \\
& \frac{1}{2} \cdot 7.920 \\
& =30.875 \mathrm{~kg} \\
& =3.150 \mathrm{~kg} \\
& =59.400 \mathrm{~kg} \\
& =3.960 \mathrm{~kg} \\
& \overline{114.097 \mathrm{~kg}} \\
& \sigma_{2}=\sigma_{3}=\frac{114.097}{350 \cdot 50}=6,52<7,77 \mathrm{kp} / \mathrm{cm}^{2} \text { (válido) }
\end{aligned}
$$

No hubiese sido preciso reforzar, pues, la pilastra.

\section{BIBLIOGRAFIA}

1) "Dissesti statici delle strutture edilizie». Sisto Mastrodicasa. Sttima edizione. Ed. Ulrico Hoepli. Milano.

2) "Ossatures des batiments". Andre Coin. Editions Eyrlles. París, 1973.

3) NTE-103 (EFL).

4) $\mathrm{EH}-80$. art. 53.
5) "Estructuras metálicas de edificios». Altos Hornos de Vizcaya (J. Batanero, R. Rodríguez Borlado, C. Martínez Lasheras, C. Moras Zancajo). 1971.

6) Catálogos Freyssinet.

7) "El cálculo de las placas de anclaje de soportes metálicos». M. Soto Pardo. Informes de la Construcción n. ${ }^{\circ} 230$, mayo 1971. Madrid.

\section{publicación del I.E.T.c.c.}

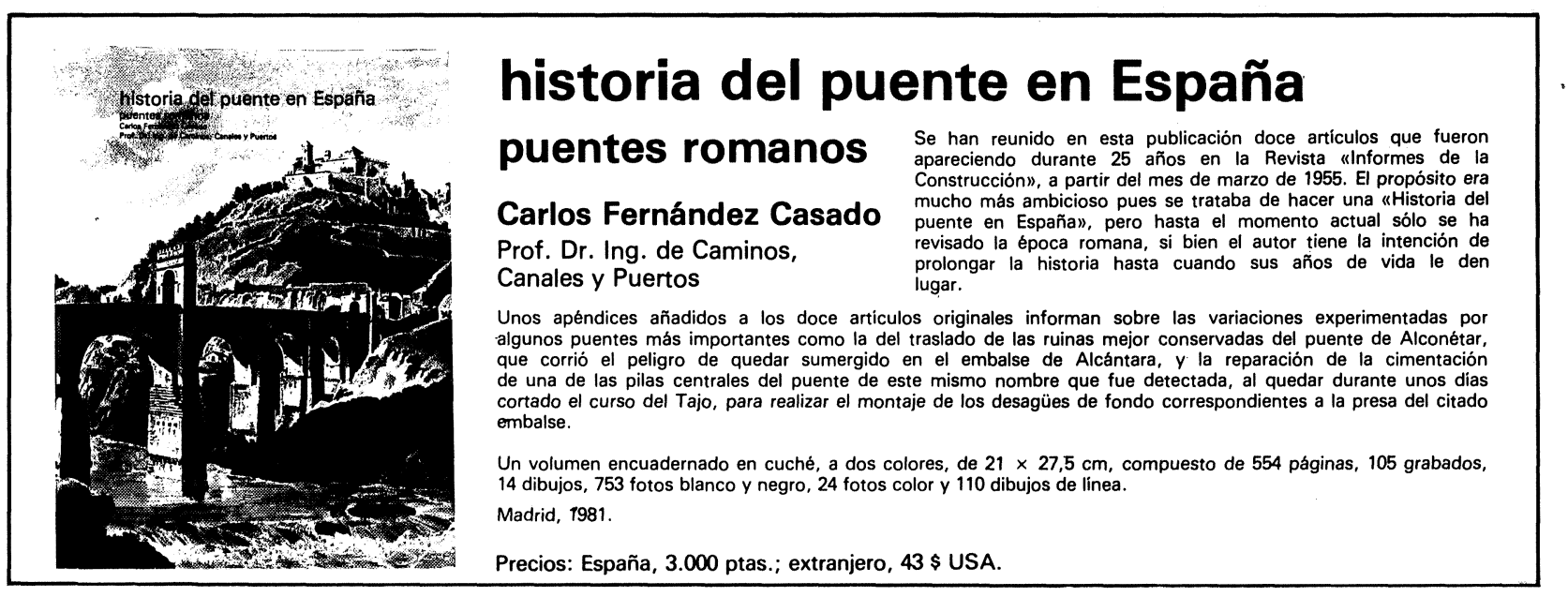

